Quim. Nova, Vol. 35, No. 8, 1522-1526, 2012

\title{
UDP- $N$-ACETILGLICOSAMINA ENOLPIRUVIL TRANSFERASE: DETERMINAÇÃO DOS ESTADOS DE PROTONAÇÃO DE RESÍDUOS DE AMINOÁCIDOS DO SÍTIO ATIVO PELO MÉTODO PMG
}

\author{
Anivaldo Xavier de Souza \\ Colégio Técnico da Universidade Federal Rural do Rio de Janeiro, BR465, km 7, 23890-000 Seropédica - RJ, Brasil \\ Carlos Mauricio R. Sant'Anna* \\ Departamento de Química, Instituto de Ciências Exatas, Universidade Federal Rural do Rio de Janeiro, BR465, km 7, 23890-000 \\ Seropédica - RJ, Brasil \\ Recebido em 16/10/11; aceito em 26/3/11; publicado na web em 26/6/12

\begin{abstract}
UDP- $N$-ACETYLGLUCOSAMINE-ENOLPYRUVYL TRANSFERASE: DETERMINATION OF PROTONATION STATE OF ACTIVE SITE AMINOACID RESIDUES BY PM6 METHOD. UDP- $N$-acetylglucosamine-enolpyruvyl transferase (MurA) catalyzes the reaction between phosphoenol pyruvate and UDP-N-acetylglucosamine. We present a theoretical approach using the semiempirical PM6 method for defining protonation state of three active site residues, K22, H125, and K160. Prior comparison with neutron diffraction data showed that PM6 accurately predicted protonation states of active site residues of $\beta$-trypsin and $D$-xylose isomerase. Using the same methodology with MurA crystallographic data, we conclude that when reaction intermediate is located at the active site, H125 and K22 are in protonated form and K160 in neutral form.
\end{abstract}

Keywords: UDP-N-acetylglucosamine-enolpyruvyl transferase; reaction mechanism; semiempirical method.

\section{INTRODUÇÃO}

As enzimas UDP- $N$-acetilglicosamina enolpiruvil transferase (MurA; EC 2.5.1.7) e 5-enolpiruvil chiquimato-3-fosfato sintase (EPSP sintase, AroA; EC 2.5.1.19) pertencem à mesma família. As reações químicas catalisadas pela MurA e AroA são essenciais para bactérias. Além disso, as reações químicas catalisadas pela AroA também têm importância essencial para plantas, fungos e algas. Por isso, a inibição destas enzimas tem importantes aplicações nas áreas de Agricultura e Saúde: a enzima AroA é alvo do inibidor glifosato (Esquema 1), o princípio ativo do herbicida mais utilizado na agricultura mundial, enquanto a MurA é inibida pela fosfomicina (Esquema 1), um antibiótico de largo espectro usado no tratamento de infecções do trato urinário; resultados recentes indicam que a fosfomicina pode ser uma opção para casos de infecções causadas por bactérias multirresistentes. ${ }^{1}$
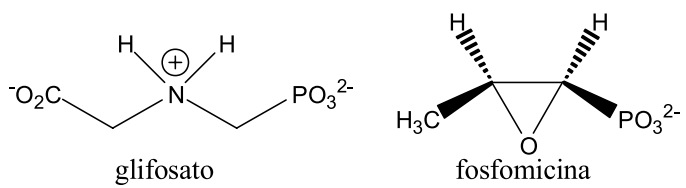

Esquema 1

Por estarem ausentes em animais, as enzimas MurA e Aroa são alvos interessantes para desenvolvimento de substâncias seletivas para o controle de bactérias. Este desenvolvimento pode ser beneficiado pelo conhecimento do mecanismo das reações catalisadas pelas enzimas MurA e Aroa. A enzima MurA catalisa o primeiro passo na biossíntese do heteropolímero peptideoglicano, o principal elemento estrutural formador da parede celular das bactérias. ${ }^{2-4}$ No processo catalisado pela MurA, ocorre a reação entre o UDP- $N$ acetilglicosamina (UNAG) e o fosfoenolpiruvato (PEP), formando os produtos enolpiruvil-UDP- $N$-acetilglicosamina (EP-UNAG) e fosfato inorgânico. ${ }^{2}$ A enzima AroA catalisa a sexta etapa da

*e-mail: santanna@ufrrj.br biossíntese do corismato, que é um precursor para a biossíntese de compostos aromáticos em plantas, fungos, bactérias e algas. ${ }^{5}$ Nesta sexta etapa ocorre a reação entre o chiquimato-3-fosfato (S3P) e o fosfoenolpiruvato (PEP) formando o produto 5- enolpiruvil chiquimato-3-fosfato (EPSP). Os mecanismos das reações catalisadas pela MurA e AroA ainda não são completamente conhecidos, porém há propostas para esses mecanismos que foram construídas a partir de observações experimentais. ${ }^{6-9}$ Estudos sugerem que essas reações ocorrem em duas etapas, sendo a primeira de adição e a segunda de eliminação, com a formação de um intermediário tetraédrico (IT) entre elas (Esquemas 2a e 2b). Entretanto, ainda não foi esclarecido de forma inequívoca quais são os aminoácidos que atuam como ácido e base em cada etapa. Neste ponto, métodos computacionais podem ser explorados para auxiliar no esclarecimento do mecanismo.

O estudo de mecanismos de reações que envolvem catálise ácida e básica depende da definição correta dos estados de protonação de resíduos do sítio ativo da enzima, que variam de acordo com o $\mathrm{pH}$ local nas proximidades de cada resíduo. Estruturas cristalográficas de proteínas geralmente não contêm informações sobre a presença de átomos de hidrogênio e não podem ser usadas diretamente na definição dos estados de protonação dos resíduos. O uso de métodos teóricos surge como uma possibilidade a ser explorada na busca da definição de estados de protonação em proteínas obtidas por cristalografia de raios-X.

Abordamos em um trabalho anterior a definição do estado de protonação dos resíduos básicos (histidinas e lisinas) do sítio ativo da AroA. ${ }^{10}$ No trabalho atual, são apresentados resultados da simulação computacional dos estados de protonação de resíduos básicos localizados no sítio ativo da enzima MurA, contendo o IT. O número elevado de átomos presentes inviabiliza a utilização de cálculos de primeiros princípios para avaliar todos os estados de protonação possíveis. Por isso, optamos por utilizar o método semiempírico. Os resultados são comparados com a estrutura cristalográfica da enzima MurA de Enterobacter cloacae, depositada no Protein Data Bank (PDB) com o código 1Q3G, que apresenta mutação de um resíduo do sítio ativo, D305, para uma alanina. ${ }^{8}$ 


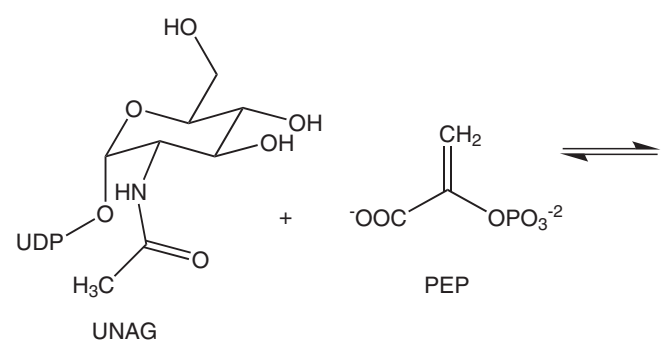

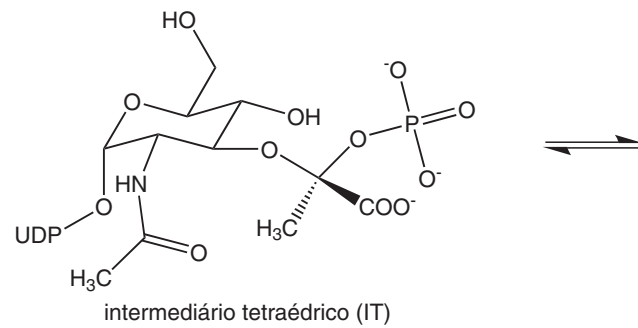

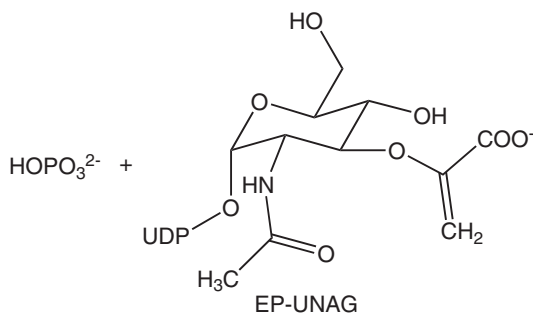

Esquema 2a. Etapas do mecanismo catalisado pela enzima MurA<smiles>C=CC#CC(=O)OC(=O)O[Na]</smiles><smiles>C[C@@](O)(O[O+])O[C@@H]1CC(C(=O)[O-])=C[C@H]([18O])[C@@H]1O</smiles><smiles>C=C([18OH])O[C@H]1CC(C(=O)[O-])=C[C@H]([18O])[C@H]1O</smiles>

Esquema 2b. Etapas do mecanismo catalisado pela enzima AroA

\section{PARTE EXPERIMENTAL}

Em trabalho anterior, ${ }^{10}$ mostramos a eficiência para prever os estados de protonação de resíduos básicos do sítio ativo de enzimas de outro método semiempírico, PM3. ${ }^{11}$ A mais recente parametrização do método semiempírico, o método PM6, tem a mesma estrutura do
PM3, porém avança na descrição das interações de caroço, no número de átomos parametrizados e prevê, com maior precisão, a geometria e o calor de formação de compostos de interesse bioquímico. ${ }^{12,13} \mathrm{O}$ erro médio para a previsão de comprimentos de ligação para compostos contendo os elementos mais comuns em compostos orgânicos, $\mathrm{C}, \mathrm{H}$, $\mathrm{N}, \mathrm{O}, \mathrm{F}, \mathrm{Cl}, \mathrm{Br}, \mathrm{I}, \mathrm{S}$ e P, é de apenas $0,031 \AA \AA^{12}$ Por isso, decidimos explorar esse método neste trabalho, mas antes realizou-se um teste para avaliar a eficiência do método PM6 na previsão de estados de protonação de aminoácidos básicos em proteínas, comparando os resultados obtidos com esse método semiempírico com dados de difração de nêutrons das proteínas $D$-xilose isomerase, com resolução de $2,2 \AA$, e $\beta$-tripsina, com resolução de $1,8 \AA$, depositadas no Protein Data Bank com os códigos 2 GVE e 1NTP, respectivamente. ${ }^{14,15} \mathrm{~A}$ técnica de espalhamento de nêutrons é um método que permite localizar átomos de hidrogênio pela introdução de deutério na proteína, identificando-se quais átomos de hidrogênio são trocados por deutério e a extensão da troca.

Três arquivos de entrada para os cálculos foram construídos a partir das estruturas $2 \mathrm{GVE}$ e $1 \mathrm{NTP}$, utilizando-se o programa Rasmol 2.6. ${ }^{16} \mathrm{Um}$ dos arquivos foi construído selecionando-se os resíduos de aminoácidos D101, T105, A106, N107, G142, A143, A148, K149, D150, V151 e A154 e três moléculas de água em contato direto com esses aminoácidos, a partir da estrutura da $D$-xilose isomerase. O segundo arquivo foi construído a partir da estrutura da enzima $\beta$-tripsina, selecionando-se os resíduos H57, A55, A56, C58, Y59, Y94, L99, D102, S195 e S214 e um ligante cocristalizado, o éster monoisopropílico do ácido fosfônico (MIP). $\mathrm{O}$ terceiro arquivo de entrada também foi construído a partir da estrutura da enzima $\beta$-tripsina, selecionando-se os resíduos $\mathrm{H} 40$, S32, L33, N34, Y39, F41, C42, G43, I73, W141 e G193. Para a transformação de coordenadas dos arquivos de dados foi utilizado o programa Babel 1.6. ${ }^{17}$

Os estados de protonação dos resíduos de aminoácidos básicos do sítio ativo da enzima MurA de Enterobacter cloacae foram avaliados usando-se a estrutura cristalográfica dessa enzima, obtida através de raios-X e depositada no PDB sob o código 1Q3G. ${ }^{8}$ Foram selecionados os aminoácidos e as moléculas de água (14) com ao menos um átomo situado em uma esfera de 6,0 $\mathrm{A}$ ao redor do intermediário da reação. Os aminoácidos selecionados foram: K22, N23, L26, P27, D49, R91, A92, W95, C115, I117, A119, R120, P121, V122, D123, L124, H125, I126, K160, V161, S162, V163, G164, A165, E188, R232, I233, P303, T304, A305, T326, I327, F328, E329, R331, L370, R371 e R397. Após a seleção dos aminoácidos e moléculas de água, foi usado o programa Babel 1.6 para adicionar os átomos de hidrogênio e para conversão de coordenadas. Os aminoácidos, o IT e as moléculas de água, todos com seus respectivos hidrogênios, somaram cerca de 800 átomos.

Nessa seleção, há três resíduos de aminoácidos básicos cujos estados de protonação podem variar de acordo com o pH local, H125, K22 e K160. Permutando-se os estados de protonação desses três resíduos de aminoácidos, foram obtidas todas as possíveis combinações dos estados de protonação, num total de oito estruturas. Em todas as estruturas os resíduos de arginina foram considerados protonados (catiônicos) enquanto os resíduos de aspartato e glutamato foram considerados na forma aniônica. O efeito da água como solvente foi incluído através do método COSMO durante o procedimento de minimização de energia. ${ }^{18}$

As estruturas foram otimizadas com o programa Mopac2009, ${ }^{19}$ com a rotina para otimização de geometria EF (Eigenvector Following) até um gradiente $<1,0 \mathrm{kcal} /(\AA$ ou rad). Pelo grande número de orbitais associados às estruturas, foi usado o método de escalonamento linear MOZYME que reduz significativamente o tempo de cálculo pelo uso de orbitais moleculares localizados. ${ }^{20}$ 


\section{RESULTADOS E DISCUSSÃO}

\section{Comparação dos resultados do método PM6 e dos dados obtidos por difração de nêutrons}

O desempenho do método PM6 na avaliação de estados de protonação foi analisado através de algumas distâncias que estão associadas a importantes interações das estruturas. Para a análise dos resultados obtidos com a $D$-xilose isomerase, foram escolhidas as distâncias que mostram a interação do átomo $\mathrm{N}^{\zeta}$ da cadeia lateral de K149 com os resíduos de aminoácidos T105, D101 e N107 (Tabela 1).

Tabela 1. Comparação da estrutura otimizada com o método PM6 com dados de difração de nêutrons da enzima $D$-xilose isomerase (código no PDB 2GEV)

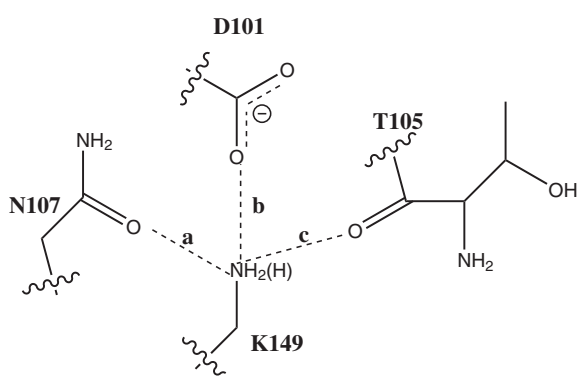

\begin{tabular}{cccccc}
\hline $\begin{array}{c}\text { Distâncias } \\
(\AA)\end{array}$ & $\begin{array}{c}\text { Experi- } \\
\text { mental }\end{array}$ & $\begin{array}{c}\text { K149 } \\
\text { protonada }\end{array}$ & $\begin{array}{c}\text { K149 } \\
\text { neutra }\end{array}$ & $\begin{array}{c}\text { K149 } \\
\text { neutra }\end{array}$ & $\begin{array}{c}\text { K149 } \\
\text { neutra }\end{array}$ \\
\hline a & 2,58 & $\mathbf{2 , 8 8}$ & 3,78 & 2,99 & 5,37 \\
b & 2,98 & $\mathbf{2 , 7 2}$ & 3,08 & 4,18 & 3,57 \\
c & 2,99 & $\mathbf{2 , 6 8}$ & 3,09 & 2,88 & 3,33 \\
DMQ* & - & $\mathbf{0 , 2 9}$ & 0,70 & 0,73 & 1,66 \\
\hline
\end{tabular}

*Desvio médio quadrático das diferenças entre os valores experimentais e calculados.

A Tabela 1 apresenta os resultados obtidos com o resíduo K149 no estado protonado (catiônico) e em três estados neutros, que correspondem a três diferentes orientações do grupo amino em relação aos outros resíduos de aminoácido. Somente quando o resíduo K149 está protonado, todas as três distâncias medidas se aproximam dos valores experimentais, resultando no menor desvio médio quadrático (DMQ) dentre os estados avaliados. As estruturas otimizadas com o método PM6, portanto, sugerem que o resíduo K149 está protonado, o que está de acordo com o número de hidrogênios ligados ao nitrogênio da cadeia lateral, identificados pelo método de difração de nêutrons. ${ }^{14}$

Para a análise dos resultados obtidos com os dois modelos construídos a partir da estrutura experimental da $\beta$-tripsina (1NPT) foram escolhidas as distâncias representadas na Tabela 2. No primeiro modelo, a corresponde à distância entre H57 e o ligante MIP, enquanto b corresponde à distância entre H57 e D102. No segundo modelo, c corresponde à distância entre a H40 e o G193, enquanto d corresponde à distância entre H40 e a S32.

Essas histidinas foram escolhidas por estarem próximas de grupos com caráter aceptor de ligação hidrogênio. Histidinas são particularmente sensíveis ao $\mathrm{pH}$ local, podendo apresentar-se na mesma estrutura nos estados neutros e catiônicos.

A Tabela 2 mostra os resultados dos modelos otimizados com os resíduos $\mathrm{H} 57$ e $\mathrm{H} 40$ no estado cationico ( $\mathrm{N}$ e $\mathrm{N}$ protonados) e dois estados neutros com apenas um dos nitrogênios protonados. Podese observar nessa tabela que, quando H57 está no estado catiônico, as distâncias avaliadas são mais curtas e mais próximas daquelas obtidas experimentalmente por difração de nêutrons, ${ }^{15}$ resultando em um DMQ bem menor para as diferenças em relação aos valores experimentais. De forma semelhante, quando His40 está no estado catiônico, as distâncias são menores e mais próximas dos valores obtidos por difração de nêutrons, ${ }^{15}$ novamente levando a um DMQ das diferenças dos valores experimentais mais baixo do que para os estados neutros. Os resultados alcançados com o método PM6 indicam que as duas histidinas devem se encontrar no estado catiônico, em acordo com o número de hidrogênios associados a estes resíduos, identificados por difração de nêutrons. $\mathrm{O}$ conjunto dos resultados indica que o uso de critérios de distância entre átomos que participam de ligações hidrogênio em estruturas modeladas com o método PM6 é adequado para determinar os estados de protonação em resíduos de lisina e de histidina nestas proteínas.

\section{Estados de protonação do sítio ativo da MurA}

A seguir, avaliou-se o estado de protonação dos resíduos de histidina e lisina no sítio ativo da enzima MurA. A Tabela 3 mostra as combinações dos três resíduos de aminoácidos do sítio ativo da MurA que tiveram seus estados de protonação avaliados, gerando oito estruturas que foram otimizadas com o método PM6. A análise dos estados de protonação dos resíduos K22, K160 e H125 da enzima MurA foi realizada através da sobreposição das estruturas otimizadas com o método PM6 com a estrutura experimental e da comparação das distâncias associadas a importantes interações destes aminoácidos com o IT e com o resíduo N23, descritas na Tabela 3.

Tabela 2. Comparação da estrutura otimizada com o método PM6 com dados de difração de nêutrons da $\beta$-tripsina (código no PDB 1NPT)
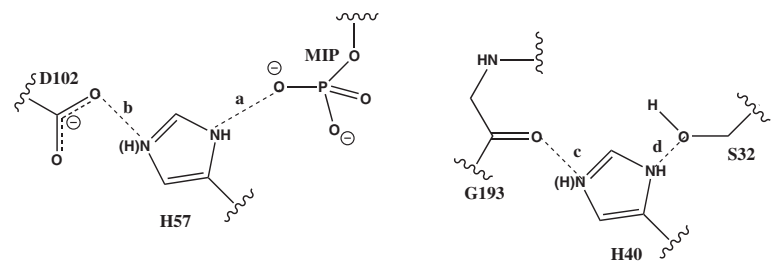

\begin{tabular}{cccccccc}
\hline Distância $(\AA)$ & Experimental & H57 protonada & H57 neutra $\left(\mathrm{N}^{\varepsilon}\right)$ & H57 neutra $\left(\mathrm{N}^{\delta}\right)$ & H40 protonada & H40 neutra $(\mathrm{N})$ & H40 neutra $(\mathrm{N})$ \\
\hline a & 2,63 & $\mathbf{2 , 6 0}$ & 2,81 & 5,65 & - & - & - \\
b & 2,48 & $\mathbf{2 , 7 2}$ & 3,38 & 2,79 & - & - & - \\
c & 2,67 & - & - & - & $\mathbf{2 , 7 3}$ & 2,80 & 3,16 \\
d & 2,59 & - & - & - & $\mathbf{2 , 7 5}$ & 2,88 & 3,38 \\
DMQ* & - & $\mathbf{0 , 1 7}$ & 0,65 & 2,15 & $\mathbf{0 , 1 2}$ & 0,22 & 0,66 \\
\hline
\end{tabular}

*Desvio médio quadrático das diferenças entre os valores experimentais e calculados. 
Tabela 3. Comparação das estruturas otimizadas com o método PM6 para os diferentes estados de protonação de resíduos do sítio ativo da enzima MurA com dados de difração de raios-X (código no PDB 1NPT)

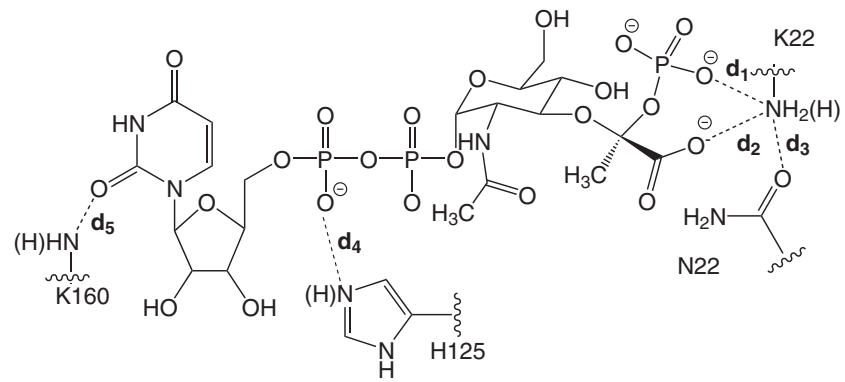

\begin{tabular}{|c|c|c|c|c|c|c|c|c|}
\hline \multirow[b]{2}{*}{ estrutura } & \multicolumn{3}{|c|}{ Estados de protonação* } & \multicolumn{5}{|c|}{ Distância $(\AA)$} \\
\hline & $\mathrm{H} 125$ & $\mathrm{~K} 22$ & K160 & $\mathbf{d}_{1}$ & $\mathbf{d}_{2}$ & $\mathbf{d}_{3}$ & $\mathrm{~d}_{4}$ & $d_{5}$ \\
\hline 1 & $\mathrm{P}$ & $\mathrm{P}$ & $\mathrm{P}$ & 2,67 & 2,99 & 2,77 & 5,43 & 2,56 \\
\hline 2 & $\mathrm{~N}$ & $\mathrm{P}$ & $\mathrm{P}$ & 2,70 & 3,04 & 2,78 & 6,42 & 2,56 \\
\hline 3 & $\mathrm{P}$ & $\mathrm{N}$ & $\mathrm{P}$ & 3,26 & 4,34 & 3,86 & 5,58 & 2,55 \\
\hline 4 & $\mathrm{~N}$ & $\mathrm{~N}$ & $\mathrm{P}$ & 3,07 & 6,02 & 4,57 & 5,77 & 2,54 \\
\hline 5 & $\mathrm{P}$ & $\mathrm{P}$ & $\mathrm{N}$ & 2,68 & 2,97 & 2,76 & 4,73 & 3,31 \\
\hline 6 & $\mathrm{~N}$ & $\mathrm{P}$ & $\mathrm{N}$ & 2,70 & 3,03 & 2,77 & 6,38 & 3,29 \\
\hline 7 & $\mathrm{P}$ & $\mathrm{N}$ & $\mathrm{N}$ & 3,05 & 6,33 & 5,42 & 5,41 & 3,90 \\
\hline 8 & $\mathrm{~N}$ & $\mathrm{~N}$ & $\mathrm{~N}$ & 3,04 & 6,45 & 5,28 & 5,57 & 3,89 \\
\hline experimental & & & & 2,82 & 2,97 & 2,64 & 4,92 & 3,20 \\
\hline
\end{tabular}

*P, protonado (catiônico); N, neutro

O valor da distância experimental que envolve a cadeia lateral do resíduo K160 é melhor reproduzido quando este resíduo é modelado no estado neutro (estruturas 5, 6, 7 e 8). Isto sugere que no momento de formação do IT o resíduo K160 se encontra neutro. Por outro lado, quando o resíduo K22 está neutro, as distâncias medidas entre o grupo amino da sua cadeia lateral e grupos negativamente carregados do IT, além do resíduo N23, são bem maiores do que os valores experimentais. Isto é um indicativo que o resíduo K22 está protonado, o que ocorre nas estruturas 1, 2, 5 e 6 . A intercessão entre os dois conjuntos é as estruturas 5 e 6 . Dentre estas estruturas, a distância medida entre o resíduo $\mathrm{H} 125$ e o grupo fosfato está mais próxima do valor experimental quando o resíduo se encontra catiônico, o que ocorre apenas na estrutura 5. O conjunto de resultados indica que a estrutura 5 representa melhor a estrutura experimental. Em resumo, a análise dos resultados sugere que, no momento de formação do IT, os resíduos de aminoácidos H125 e K22 estão protonados e o resíduo K160 está neutro.

\section{CONCLUSÃO}

$\mathrm{Na}$ análise comparativa com estruturas experimentais contendo as posições de átomos de hidrogênio, os resultados teóricos obtidos com o método PM6 indicam que o resíduo de aminoácido K149 da enzima $D$-xilose isomerase (código $2 \mathrm{GEV}$ no $\mathrm{PDB}$ ) e os resíduos H57 e H40 da $\beta$-tripsina (1NTP) estão todos no estado protonado, em perfeita concordância com os resultados experimentais obtidos por difração de nêutrons. Esses resultados nos permitem concluir que o uso de critérios de distâncias interatômicas calculadas com o método PM6, da mesma forma que com o método PM3 avaliado em um trabalho anterior de nosso grupo, é adequado para prever estados de protonação de resíduos de aminoácidos em proteínas.

A aplicação do mesmo método na avaliação dos estados de protonação dos resíduos de aminoácidos H125, K22 e K160 da enzima MurA indica que o resíduo H125 está protonado no momento da formação do IT; os dois resíduos de lisina são previstos em estados de protonação diferentes: K160 está neutra e K22, protonada. K160 teria então uma função apenas estrutural, auxiliando na orientação do fragmento de UNAG. Já o resíduo K22 seria a base na etapa de adição, possivelmente desprotonando o grupo 3'-OH da UNAG e tornando-o um melhor nucleófilo para se adicionar ao PEP, o que corrobora sugestões da literatura baseadas na interpretação de dados cristalográficos da enzima MurA e na comparação com dados cinéticos da enzima AroA.$^{6-8}$ Além disso, a carga positiva do resíduo K22 localizada próxima do fragmento de PEP do IT, negativamente carregado, deve ser um dos fatores que contribuem para estabilizar o IT no interior da enzima através de interações íon-íon (auxiliado pelo grande número de resíduos de arginina próximos, também carregados positivamente). Por limitações estruturais, o resíduo H125 protonado não é capaz de se aproximar o suficiente do grupo difosfato do IT para fazer interações íon-íon efetivas; o seu efeito deve ser, portanto, um efeito de estabilização à distância sobre o IT (efeito de campo).

Deve ser destacado que nossos resultados são baseados em uma estrutura cristalográfica que reflete um momento do mecanismo enzimático, e que estes estados podem se modificar durante o decorrer do mecanismo. Estas informações devem, contudo, ser úteis no planejamento estrutural de inibidores enzimáticos que mimetizem o IT (ou mesmo o estado de transição que o precede) para a enzima MurA.

\section{REFERÊNCIAS}

1. Falagas, M. E.; Kastoris, A. C.; Kapaskelis, A. M.; Karageorgopoulos, D. E.; Lancet Infect. Dis. 2010, 10, 43.

2. Marquardt, J. L.; Brown, E. D.; Lane, W. S.; Haley, T. M.; Ichikawa, Y.; Wong, C. H.; Walsh, C. T.; Biochemistry 1994, 33, 10646.

3. van Heijenoort, J. Em Bacterial Cell wall; Ghuysen, J. M.; Hakenbeck R., eds.; Elsevier Science Publishers B.V: Amsterdam, 1994, chap. 3.

4. Schonbrunn, E.; Sack, S.; Eschenburg, S.; Perrakis, A.; Krekel, F.; Amrhein, N.; Mandelkow, E.; Structure 1996, 4, 1065. 
5. Roberts, F.; Roberts, C. W.; Johnson, J. J.; Kyle, D. E.; Krell, T.; Coggins, J. R.; Coombs, G. H.; Milhous, W. K.; Tzipori, S.; Ferguson, D. J.; Chakrabarti, D.; Mcleod, R.; Nature 1998, 393, 801.

6. Mizyed, S.; Wright, J. E. I.; Byczynski, B. P.; Berti, J.; Biochemistry 2003, 42, 6986.

7. An, M.; Maitra, U.; Neidlein, U.; Bartlett, P. A.; J. Am. Chem. Soc. 2003, $125,12759$.

8. Eschenburg, S.; Kabsch, W.; Healys, M. L.; Schönbrunn, E.; J. Biol. Chem. 2003, 278, 49215.

9. Berti, P. J.; Chindemi, P.; Biochemistry 2009, 48, 3699.

10. Souza, A. X.; Sant'Anna, C. M. R.; Bioorg. Chem. 2008, 36, 113.

11. Stewart, J. J. P.; J. Comp. Chem. 1989, 10, 209.

12. Stewart, J. J. P.; J. Mol. Modeling 2007, 13, 1173.

13. Morgon, N. H.; Coutinho, K.; Métodos de Química Teórica e Modelagem Molecular, Ed. Livraria da Física: São Paulo, 2007.
14. Katz, A. K.; Li, X.; Carrell, H. L.; Hanson, B. L.; Langan, P.; Coates, L.; Schoenborn, B. P.; Glusker, J. P.; Bunick, G. J.; Proc. Nat. Acad. Sci. U.S.A. 2006, 103, 8342.

15. Kossiakoff, A. A.; Basic life sciences 1984, 27, 281.

16. Sayle, R.; Glaxo Wellcome Research and Development, Stevenage, 1993.

17. Walters, P.; Stahl, M.; Babel 1.6; Tucson, Az. University of Arizona, 1996.

18. Klamt, A.; Schüümann, G.; J. Chem. Soc., Perkin Trans. 2: Phys. Org. Chem. 1993, 5, 799.

19. MOPAC2009, Stewart, J. J. P.; Stewart Computational Chemistry, Version $10.153 \mathrm{~W}$ web: http://OpenMOPAC.net.

20. Stewart, J. J. P.; Int. J. Quant. Chem. 1996, 58, 113. 